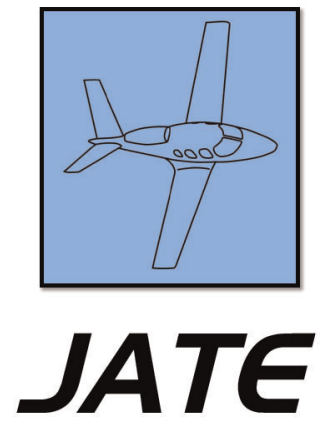

Journal of Aviation Technology and Engineering 7:1 (2017) 45-49

\title{
Assessing the Benefits of Performance-Based Navigation Procedures
}

\author{
Kabir O. Kasim
}

Embry-Riddle Aeronautical University

\begin{abstract}
Performance-based Navigation (PBN) allows aviation operations to be conducted based on actual operational requirements rather than the requirements of ground-based equipment. Although the general operational benefits of PBN procedures have been recognized by various studies, there is a need to specify the actual benefits in terms of the frequency of event anomalies that could be expected from the use of PBN procedures. The study reviewed some of the available literature and identified some operational improvements as reported by previous authors. The study then proceeded to review archival data from the Aviation Safety Reporting System (ASRS) database with a view to identifying the link between the use of PBN procedures and reported event anomalies. Overall, there were significantly fewer reported event anomalies when PBN procedures were used than when PBN procedures were not used. It is suggested that air operators and air navigation service providers conduct appropriate risk and safety assessments in their consideration of an increased use of PBN procedures.
\end{abstract}

Keywords: performance-based navigation, event anomalies

\section{Introduction}

The use of Performance-based Navigation $(\mathrm{PBN})$ techniques has been adopted by the International Civil Aviation Organization (ICAO) as a solution to some of the challenges of the conventional ground-based navigation aids. PBN is a generic term that refers to a system of navigation that allows navigation requirements to be based on operational requirements. With PBN, navigational specifications identify the sensors and equipment required to meet the performance requirements. ICAO has ensured that the navigation specifications are defined at a sufficient level of detail to facilitate global harmonization by providing specific implementation guidance for states and operators (ICAO, 2008).

Guidance from the Federal Aviation Administration (FAA) clarifies PBN and the relationship to area navigation (RNAV) and required navigation performance (RNP). RNAV enables aircraft to fly on any desired flight path within the coverage of appropriate ground or space-based navigation aids, within the limits of the capability of the aircraft's self-contained systems, or a combination of both capabilities. RNP is RNAV with the addition of on-board performance monitoring and alerting capability. RNP operations allow the aircraft navigation system to monitor the navigation performance it achieves and inform the crew if the requirement is not met during an operation. The performance requirements of PBN are conveyed to the operators through navigation specifications. PBN navigation specifications include RNAV 1, RNP 1, RNAV 2, RNAV 5, RNP 10, and RNP 4, as well as RNAV (GPS) and RNAV (RNP) approaches (FAA, n.d.). 
Butchibabu, Midkiff, Kendra, Hansman, and Chandra (2010) noted in their study that PBN procedures offered significant safety improvements. Although they stated that PBN procedures allow operators to benefit from new levels of flexibility as it pertains to terrain, airspace, and environmental considerations, they also accept that PBN procedures "create both opportunity and challenges" (p. 4). These benefits were described further by Muller, Uday, and Marais (2011). They noted that, in terms of the terrain, PBN procedures help improve access and increase safety margins associated with high-terrain airports and remote areas with little ground-based navigation infrastructure. In addition, they highlighted that the airspace improvements are seen in reduced congestion and the safe reduction in separation, while environmental considerations include reduced fuel burn, reduced harmful emissions, and reduced noise pollution.

In their consideration of PBN benefits, Mayer, Crow, Zondervan, and Allerdice (2015) looked at PBN procedures specifically as they relate to independent final approaches for landing on parallel runways. They stated that the use of published PBN procedures has resulted in improvements in navigational precision and "improved the reliability and repeatability of operations in terminal airspace surrounding major United States (U.S.) airports" (p. 1). Their conclusion was that there were potential benefits relating to "improvements at airports with less than widely spaced runways, enhancements in operational safety, and reductions in approach monitoring needs" (p. 10).

The purpose of this study is to identify whether there is a relationship between the use of PBN procedures and reported event anomalies. The study will compare reported safety issues when PBN procedures are used against when they are not used. The study also seeks to update the analysis by Butchibabu et al. (2010) by reviewing more recent reports from the Aviation Safety Reporting System (ASRS) database and present lessons learned from the use of PBN. The ASRS database was selected due to the confidential nature of the system and the belief that voluntary reports filed without unnecessary pressures on reporters should result in valuable data for the aviation community.

The organization of the remaining sections of this paper is as follows: The method section describes the mode of data collection including the criteria used to search the ASRS database. Next, the results section presents the event anomalies and the number of times each event occurred, while the discussion section presents an analysis of the results. Brief concluding remarks are then provided followed by a listing of all applicable references. Finally, the acronyms used in the paper are listed in the appendix.

\section{Method}

This study is based on archival data from the ASRS database. The following fields from the database were specified to obtain reports relevant to the study: Date of Incident, Flight Conditions, Flight Phase, Location, and Event Type. The search criteria and the text keywords used in narratives and synopses are listed in Table 1.

The criterion used to select the locations was the number of PBN procedures available at the location. The ten airports selected had twenty or more PBN procedures including RNAV STARs, RNAV SIDs, and RNP AR approaches. Based on the search criteria, a total of 377 reports were retrieved in Microsoft Excel format. The narratives from the reports were reviewed individually in the attempt to identify those reports associated with the use of PBN procedures.

\section{Results}

The results showed that there were 184 event anomalies associated with the use of PBN procedures while there were 297 event anomalies when there were no associated PBN procedures in use. The total number of event anomalies reported is greater than the number of actual reports because it is possible for one report to contain more than one event anomaly. The frequency of the event anomalies with and without the use of PBN procedures is shown in Table 2.

Although the data showed that there were more altitude and track deviation events when PBN procedures were in use than when they were not, the overall number of event anomalies related to deviations (including altitude, track, and procedural) was less than the number of events when PBN procedures were not in use.

Table 1

ASRS fields and search criteria.

\begin{tabular}{ll}
\hline Field & Search Criteria \\
\hline Date of Incident & January 1, 2010-December 31, 2015 \\
Flight Conditions & IMC, Marginal, Mixed, VMC \\
Flight Phase & Takeoff, Initial Climb, Climb, Cruise, Descent, Initial Approach, Final Approach, Landing \\
Location & DEN, MEM, ATL, PHX, STL, BNA, CVG, MIA, IAH, ABQ \\
Event Type & Airspace Violation, ATC Issues, Conflict (Airborne, NMAC), Deviation (Altitude, Procedural, Speed, Track/Heading), \\
& In-flight Event (CFTT/CFIT), Loss of Aircraft Control (Unstabilized Approach) \\
Text Keywords & RNAV, GNS, GPS, STAR, SID, CHART, APPROACH, IF, IAF, FAF \\
\hline
\end{tabular}


Table 2

Frequency of event anomalies.

\begin{tabular}{lcc}
\hline Event Anomaly & PBN Procedures in Use & PBN Procedures Not in Use \\
\hline Procedural Deviation & 61 & 96 \\
Altitude Deviation & 51 & 38 \\
Track Deviation & 45 & 43 \\
Unstabilized Approach & 2 & 24 \\
Airborne Conflict & 8 & 34 \\
In-flight Event & 8 & 44 \\
ATC Issues & 9 & 18 \\
TOTAL & $\mathbf{1 8 4}$ & $\mathbf{2 9 7}$ \\
\hline
\end{tabular}

\section{Procedural Deviation}

Procedural deviations constituted most of the event anomalies in both cases of PBN use and non-PBN use. There were 61 (or $73 \%$ ) procedural deviation events reported with PBN procedures in use and 96 (or 32\%) when PBN procedures were not used. Procedural deviations occurred due to non-compliance to published operational procedures, issues with STARs, and SIDs. They also involved occurrences on the ground.

\section{Altitude Deviation}

Altitude deviations accounted for 51 (or 28\%) of events with PBN procedures in use and 38 (or 13\%) of events without the use of PBN procedures. The reported altitude deviations included cases of unauthorized descents, altitude overshoots, and failures to meet crossing restrictions. It was also noted that the deviations were a mix of VFR and IFR flights. Some specific events identified the following: a steep descent into PHX, multiple events from one aircraft type, Airbus A300, and RNAV STARs into MEM.

\section{Track Deviation}

Deviations from planned or assigned track accounted for 45 (or 25\%) of events with PBN procedures in use and 43 (or 15\%) of events without the use of PBN procedures. Track deviations usually occurred due to misunderstanding of heading clearances after departure, FMS failures, and localizer issues on approach to landing. In one particular case, heading vectors issued by ATC were in conflict with ATC issued clearance on stated RNAV departure. This event was also recorded under ATC issues.

\section{Unstabilized Approach}

There were very few reports of unstabilized approaches (two, or $0.01 \%$ ) when PBN procedures were in use. The number of reports when there were no PBN procedures in use was 24 (or $0.08 \%$ ). Unstabilized approaches are often the outcome of incorrect set-up for the approach or inappropriate use of automation.

\section{Airborne Conflict}

Event anomalies related to airborne conflicts occurred eight times (or $0.04 \%$ ) with the use of PBN procedures while there were 34 reports (or $0.11 \%$ ) when PBN procedures were not in use. Airborne conflicts occurred at various altitudes and sometimes resulted in aircrew responding to TCAS traffic advisories (TA). A particular event identified involved aircraft landing on one runway while another aircraft was cleared to land on the opposite runway at the same time.

\section{In-Flight Event}

In-flight events include occurrences due to turbulence, wake vortex, and weather. In-flight events accounted for eight (or $0.04 \%$ ) of reports when PBN procedures were in use and 44 reports (or $0.15 \%$ ) when PBN procedures were not in use. One of the more common in-flight events occurring was wake vortex due to inadequate separation of aircraft during takeoff and landing.

\section{ATC Issues}

ATC issues occurred in nine reports (or $0.05 \%$ ) when PBN procedures were in use and 18 (or $0.06 \%$ ) of reports when PBN procedures were not in use. ATC issues identified include frequency congestion, ambiguous clearances, and ground /runway incursions.

\section{Discussion}

A search of the ASRS database was conducted to determine event anomalies related to the use of $\mathrm{PBN}$ procedures. In searching the database, the use of PBN procedures was determined by the presence of the following keywords: RNAV STARs, RNAV SIDs, and RNP AR approaches. Of the 377 reports retrieved from the database, 184 event anomalies were related to the use of PBN procedures while there were 297 event anomalies when there were no associated PBN procedures in use. Other issues identified from the study concerned reports of complex RNAV arrivals at DEN, and MEM RNAV reported to be prone to pilot deviations and errors. 
The results from the study suggested that there were significantly fewer reported event anomalies with the use of PBN procedures. Although two particular event anomalies, altitude deviations and track deviations, showed a higher figure when PBN procedures were in use, the results showed that, overall, there were fewer event anomalies reported when PBN procedures were in use. This seems to suggest that some of the operational benefits reported from the use of PBN procedures could be quantified in terms of the reduction in the number of event anomalies reported.

Benefits of PBN have been reported for specific operational areas. For example, implementing RNAV SIDs at ATL resulted in tighter patterns for the departures and subsequently allowed arrival aircraft a greater choice of routes (Helfrick, 2015). Similarly, RNAV SID trials at SJC showed that a $12.5 \%$ reduction in inter-flight spacing resulted in a $45 \%$ improvement in total en route transitions' capacity (Timar, Hunter, \& Post, 2013).

The data available for the study was limited by the fact that the ASRS database contains only voluntary reports and therefore may only reveal a small portion of the events that occur. ASRS reports, being self-reports may also contain subjective biases of the individuals (Butchibabu et al., 2010). Another limitation is that the database does not contain specific categories for PBN procedures. In view of this, each report was reviewed independently to identify reports with PBN issues. Finally, the study did not consider specific human factors issues with the event anomalies reported.

\section{Summary and Conclusions}

The study has identified significant operational benefits from the use of PBN procedures. The sample of reports reviewed also revealed that there were fewer event anomalies reported with the use of PBN procedures. The FAA in its roadmap for PBN highlights that the use of RNAV and RNP is expected to: increase safety, improve airport and airspace access, enhance reliability and reduce delays, improve efficiency and flexibility, and reduce workload and improve productivity of air traffic controllers (FAA, 2006). The FAA overview mandates "RNAV everywhere in the continental United States and RNP in busy en route and terminal airspace during the period 2016-2025" (p. 11).

It is expected that the use of PBN procedures will become more prevalent, especially in areas where there are no operational limitations to their use. The increased use of
PBN procedures requires that more specific investigations of the benefits and hazards should be carried out. It is also suggested that further study could focus on identifying the factors specific to altitude deviations since this category of deviations showed a higher event tally with PBN procedures.

It is expected that aircraft operators would carry out operational safety assessments to ensure all associated risks are considered before commencing the use of PBN procedures. Regular reviews to determine benefits should also be completed. This will be in line with elements of a safety management system (SMS).

\section{References}

Butchibabu, A., Midkiff, A., Kendra, A., Hansman, R. J., \& Chandra, D. C. (2010, November). Analysis of safety reports involving area navigation and required navigation performance procedures. Paper presented at the 2010 International Conference on Human-Computer Interaction in Aeronautics (HCI-Aero), Cape Canaveral, FL. Retrieved from http://ntl.bts.gov/lib/44000/44100/44160/hciaero_1_.pdf

Federal Aviation Authority (FAA). (n.d.). Performance-based flight systems branch: Performance-based navigation $(\mathrm{PBN})$ guidance \& approval. Retrieved from https://www.faa.gov/about/office_org/ headquarters_offices/avs/offices/afx/afs/afs400/afs470/pbn/

Federal Aviation Authority (FAA). (2006). Roadmap for performancebased navigation. Version 2.0. Retrieved from https://www.faa.gov/ about/office_org/headquarters_offices/avs/offices/afx/afs/afs400/rnp/ media/RNProadmap.pdf?CFID $=82675925 \&$ CFTOKEN $=\mathrm{e} 3 \mathrm{a} 078 \mathrm{f} 224 \mathrm{~b}$ a32fe-1735E9D8-99E8-389F-7B0559AB2E80FF11\&jsessionid=73F0 497B89F080272330D4C1F99DFF38.www

Helfrick, A. (2015). The centennial of avionics: Our 100-year trek to performance-based navigation. IEEE Aerospace and Electronic Systems Magazine, 30(9), 36-45. https://doi.org/10.1109/MAES.2015.140226

International Civil Aviation Organization (ICAO). (2008). Performancebased navigation manual (Doc 9613; third edition). Retrieved from http://www.icao.int/SAM/Documents/2009/SAMIG3/PBN\% 20Manual\%20-\%20Doc\%209613\%20Final\%205\%2010\%2008\% 20with\%20bookmarks1.pdf

Mayer, R. H., Crow, B. M., Zondervan, D. J., \& Allerdice, J. K. (2015). Concept and benefits of PBN-enabled parallel approach operations. Proceedings of the 15th AIAA Aviation Technology, Integration, and Operations Conference, AIAA Aviation, (AIAA 2015-3027). https://doi. org/10.2514/6.2015-3027

Muller, D., Uday, P., \& Marais, K. (2011). Evaluation of the potential environmental benefits of RNAV/RNP arrival procedures. Proceedings of the 11th AIAA Aviation Technology, Integration, and Operations Conference, including the AIA. https://doi.org/10.2514/6.2011-6932

Timar, S., Hunter, G., \& Post, J. (2013, June). Assessing the benefits of NextGen Performance Based Navigation (PBN). In Tenth USA/ Europe Air Traffic Management Research and Development Seminar, Chicago, Illinois. Retrieved from http://www.atmseminar.org/ seminarcontent/seminar10/papers/170-timar_0122130540-final-paper4-12-13.pdf 


\section{Appendix-Acronyms}

$\begin{array}{ll}\text { ABQ } & \text { Albuquerque International Sunport } \\ \text { ASRS } & \text { Aviation Safety Reporting System } \\ \text { ATC } & \text { Air Traffic Control } \\ \text { ATL } & \text { Hartsfield-Jackson Atlanta International Airport } \\ \text { BNA } & \text { Nashville International Airport } \\ \text { CFTT/CFIT } & \text { Controlled Flight Toward Terrain/Controlled Flight Into Terrain } \\ \text { CVG } & \text { Cincinnati/Northern Kentucky International Airport } \\ \text { DEN } & \text { Denver International Airport } \\ \text { FAA } & \text { Federal Aviation Administration } \\ \text { FAF } & \text { Final Approach Fix } \\ \text { GNS } & \text { Global Navigation System } \\ \text { GPS } & \text { Global Positioning System } \\ \text { IAF } & \text { Initial Approach Fix } \\ \text { IAH } & \text { George Bush Intercontinental Airport } \\ \text { IF } & \text { Initial Fix } \\ \text { IMC } & \text { Instrument Meteorological Conditions } \\ \text { MEM } & \text { Memphis International Airport } \\ \text { MIA } & \text { Miami International Airport } \\ \text { NMAC } & \text { Near Midair Collision } \\ \text { PBN } & \text { Performance-based Navigation } \\ \text { PHX } & \text { Phoenix Sky Harbor International Airport } \\ \text { RNAV } & \text { Area Navigation } \\ \text { RNP } & \text { Required Navigation Performance } \\ \text { RNP AR } & \text { Required Navigation Performance Authorization Required } \\ \text { SID } & \text { Standard Instrument Departure } \\ \text { SJC } & \text { San Jose International Airport } \\ \text { SMS } & \text { Safety Management System } \\ \text { STAR } & \text { Standard Terminal Arrival Route } \\ \text { STL } & \text { Lambert-St. Louis International Airport } \\ \text { VMC } & \text { Visual Meteorological Conditions } \\ & \end{array}$

Kabir Kasim holds an Air Transport Pilot's License (Helicopter) and completed an MSc in air transport management. $\mathrm{He}$ is currently a $\mathrm{PhD}$ student at Embry-Riddle Aeronautical University. His research interests include human factors, pilot training, simulation, unmanned aerial systems, and performance-based navigation. 